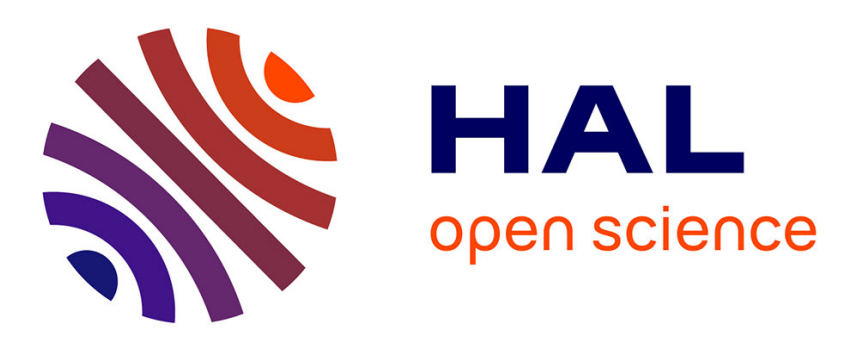

\title{
An Investigation on the Utility and Reliability of Electroencephalogram Phase Signal Upon Interpreting Cognitive Responses in the Brain: A Critical Discussion Esmaeil Seraj
}

\section{- To cite this version:}

Esmaeil Seraj. An Investigation on the Utility and Reliability of Electroencephalogram Phase Signal Upon Interpreting Cognitive Responses in the Brain: A Critical Discussion. Journal of Advanced Medical Sciences and Applied Technologies, 2016, 2. hal-02165509

\author{
HAL Id: hal-02165509 \\ https://hal.science/hal-02165509
}

Submitted on 26 Jun 2019

HAL is a multi-disciplinary open access archive for the deposit and dissemination of scientific research documents, whether they are published or not. The documents may come from teaching and research institutions in France or abroad, or from public or private research centers.
L'archive ouverte pluridisciplinaire HAL, est destinée au dépôt et à la diffusion de documents scientifiques de niveau recherche, publiés ou non, émanant des établissements d'enseignement et de recherche français ou étrangers, des laboratoires publics ou privés. 


\title{
An Investigation on the Utility and Reliability of Electroencephalogram Phase Signal Upon Interpreting Cognitive Responses in the Brain: A Critical Discussion
}

\author{
Esmaeil Seraj $^{1^{*}}$ \\ 1. Signal Processing Center, Department of Computer Science, Engineering and Information Technology, School of Electrical and Computer Engineer- \\ ing, Shiraz University, Shiraz, Iran.
}

Use yourdevice to scan
and read the article online
Cognitive Responses in Brain: A Critical Discussion. Journal of Advanced Medical Sciences and Applied Technologies (JAM-
SAT). 2016; 2(4):299-312.

Article info:

Received: 1 Sep. 2016

Accepted: 5 Nov. 2016

\section{Keywords:}

Cognitive state,

Electroencephalogram phase, EEG Phase Synchronization,

EEG Phase Desynchronization, Cognitive neuroscience

\begin{abstract}
AB STRACT
Within the neuroscience and computational neuroscience communities, applications such as evaluating different cognitive responses of the brain, brain-computer interface (BCI) systems and brain connectivity studies have increasingly been using EEG phase information during the past few decades. The utility of EEG phase can be directly linked to the neural propagation and synchronized firing of neuronal populations during different cognitive states of the brain. Nevertheless, it has previously been shown that phase of narrow-band (frequency specific) foreground EEG (desired) is prone to contain fake spikes and variations (unrelated to brain activity) in the presence of background spontaneous EEG and low SNRs of foreground EEG (the low-amplitude analytic signals or LAAS problem). Accordingly, extracting the instantaneous EEG phase sequence for further utilization upon interpreting the cognitive states of the brain using phase related quantities, such as instantaneous frequency, phase shift, phase resetting and phase locking value, is a very sensitive and rigorous process. In this study, a simple procedure is proposed to illustrate the effects of LAAS problem on the utility of EEG phase related quantities in aforementioned applications, also to investigate the reliability of interpretations of the brain's cognitive states based on such quantities. Results show that, without a proper and effective solution strategy, such potential flaws lead to incorrect physiological and pathological interpretations.
\end{abstract}

\section{Introduction}

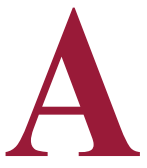

Recent scientific model proposes that functional integration is obtained within the brain due to the concept of neural assemblies. Neural assembly is known as a group of functionally interconnected neurons. The interaction between the neurons of an assembly (neuronal populations) are strong due to an amplified feedback mechanism called reentry [1]. The process of reentry requires specific timing relations, which induces neurons within an assembly to synchronize their firing rates. This has led to an increased interest in utilizing phase variables of the cerebral signals, particularly their synchronization in various applications within the area of computational and applied neuroscience [2].

\section{* Corresponding Author:}

Esmaeil Seraj, MSc.

Address: Signal Processing Center, Department of Computer Science, Engineering and Information Technology, School of Electrical and Computer

Engineering, Shiraz University, Shiraz, Iran.

Tel: +98 (912) 7756893

E-mail:e.seraj@cse.shirazu.ac.ir 
Some of these applications include the Brain-Computer Interface (BCI) systems [3, 4, 5], Event Related Potential (ERP) studies $[6,7,8]$, assessing the connectivity within brain $[9$, $10,11,2]$ and the study of brain's cognitive responses [12, $13,14]$. To use the complementary information embedded in phase of cerebral signals (over the traditional spectral amplitude), normally the Instantaneous Phase (IP) sequence is extracted and then, related quantities and features are elicited. The most popular phase-related quantities, particularly in the area of cognitive and computational neuroscience include: 1 Instantaneous Frequency (IF), 2 Phase Difference (PD), 3 Phase Shift (PS), 4 Phase Lock (PL), 5 Phase Resetting (PR) and 6 Phase-Locking Value (PLV).

The importance of phase related quantities can be linked to their vast application in many different physiological and pathological interpretations. For instance, phase differences of electroencephalogram (EEG) are used to calculate the Directional Coherence between brain records, which is a measure of the directional flow of information between two EEG electrode sites $[15,16]$. Moreover, EEG phase differences are also used in estimating the conduction velocities and synaptic integration times in different directions within brain regions $[17,18,19]$. Furthermore, phase resetting is an indicator of the dynamic balance between Event Related Synchronization (ERS) and Desynchronization (ERD) in EEG records.

As reported in previous studies, having a balanced ERS/ ERD events association is essential for normal brain function and the abnormal balance is associated with pathological conditions such as epilepsy [20,21], dementia [22, 23], autism [24] and many more [25, 26]. Additionally, phase reset in cerebral signals has been correlated to several frequency bands during different cognitive responses such as working memory [27, 28, 29], brain development [17], intelligence $[30,31]$, consciousness [32, 33, 34], sensory-motor interactions [36] and others [37, 38]. Also, phase shift events are of great interest in brain-connectivity measurements [2]. Accordingly, it can be concluded that phase-related quantities are well-known and vastly used in real-world neuroscience research and practice owing to the clear and important physiological and pathological information they provide.

The reliable calculation of phase-related variables with respect to the brain activity is highly dependent on estimating an accurate and meaningful measure of the instantaneous phase sequence of the EEG signal. Nevertheless, it has been shown that phase of narrow-band (frequency specific) foreground EEG (desired task related signal) may potentially contain fake spikes and variations (unrelated to the brain activity) in the presence of background spontaneous EEG and low SNRs of foreground EEG [39].
Accordingly, IP estimation is a very sensitive and rigorous task due to two major obstacles: 1 making the phase sequence frequency-specific and narrowing signal's spectral support as much as possible and 2 dealing with unavoidable and undeniable fake phase-jumps spread throughout the phase sequence due to the low foreground SNRs. Herein, we refer to this problem as low-Amplitude Analytic Signals (LAAS).

The problem associated with the first obstacle is that extracted phase from a wide frequency-band could be pointless [40]. For this purpose, filtering and narrowing the frequency support of EEG signal is the initial step in every phase extraction method; nevertheless, a filter with very thin Band Width (BW) or Transition Band (TB) could have very unstable behavior due to its corresponding high-order and long-impulse response (FIR filters). This instability leads to obtaining quite different phase measures with slightly changing the filter's parameters or having additional uncorrelated noise sources. Consequently, trying to have a meaningful and reliable phase sequence (narrowing the frequency support of signal as much as possible) might result in having fake information in phase of cerebral signals which are not related to the brain activity. In addition, it has been previously shown that low values of amplitude in analytic signals (foreground or desired activity) is a major source of spikes and jumps throughout the captured instantaneous phase sequence [39]. This notorious problem which is referred to as phase-slipping, leads to incorrect clinical interpretations of phase related quantities $[39,41]$.

The purpose of this study is to illustrate the effects of the Low-Amplitude Analytic Signal (LAAS) problem on the utility of the EEG phase related quantities in the mentioned applications and also to investigate the reliability of interpretations upon the brain's cognitive responses based on such quantities in order to show their potential impact on the related previous clinical studies.

In this section, first the conventional EEG phase estimation procedure is reviewed and then the basic principles underlying the LAAS issue are discussed.

\section{Conventional phase estimation method based on analytic signal}

The traditional phase extraction procedure consists of two steps including: 1Frequency filtering and narrowing signal's spectral support, and 2Estimating the phase sequence from complex representation of the filtered signal through one of the existing tools such as Fourier Transform (FT), Hilbert Transform (HT), Wavelet Transform (WT), etc.

In traditional procedures, Finite Impulse-Response (FIR) filters are widely used for the first step due to their linear 
phase-response [42, 43, 44]. For phase estimation step, it is important to choose a method which is accurate enough not to violate theoretical conditions essential for having a reliable instantaneous phase. The most commonly used method for the phase estimation is to use the analytic signal representation of the narrow-band signal [40]. Accordingly, for the signal $x(t)$, its analytical form is defined as follows [45, 46, 47]:

$$
Z-x(t)=x(t)+j H\{x(t)\}
$$

where $\mathrm{H}\{\mathrm{x}(\mathrm{t})\}$ is the Hilbert transform of $\mathrm{x}(\mathrm{t})$. Using the analytic signal representation, the Instantaneous Amplitude (IA) and instantaneous phase (IP) pair are uniquely defined as below:

$$
\begin{gathered}
a_{x}(t)=\left|z_{x}(t)\right|=\sqrt{x(t)^{2}+H\{x(t)\}^{2}} \\
\emptyset_{x}(t)=\arctan \left(\frac{H\{x(t)\}}{x(t)}\right)
\end{gathered}
$$

Unless the signal has a narrow-band spectral support, the pair $\left(\mathrm{a}_{\mathrm{x}}(\mathrm{t}), \varnothing_{\mathrm{x}}(\mathrm{t})\right)$ do not convey significant information regarding the accurate activity of the brain $[45,46]$. Accordingly, the narrow-band filtering step is essential when
EEG phase-related quantities are used to interpret cognitive states of the brain.

The Low-amplitude Analytic Signal (LAAS) problem in estimating phase of a signal

The problem of EEG IP analysis has been mainly studied within a deterministic framework, without (systematically) considering the effects of the EEG amplitude and background EEG activity in IP and IF estimation. In order to show the motivation of the current study, a synthetic signal diluted by additive noise is shown in Figure 1. The signal model is as follows:

$$
y(t)=[\bmod 0.7] \cos \left(2 \pi \int_{0}^{t} f(\tau) d \tau\right)+n(t)
$$

where $f(t)=15 \sin (2 n \times 0.8 t)+30 H z$ is the instantaneous frequency and $n(t)$ is white Gaussian noise with a variance adjusted to set the SNR of $y(t)$ to $25 \mathrm{~dB}$. According to the Figure 1, random phase jumps and instantaneous frequency (first order time derivative of instantaneous phase in Equation 3) errors concurrent with low values of analytical signal amplitude (low signal-tonoise ratios) are inevitable.
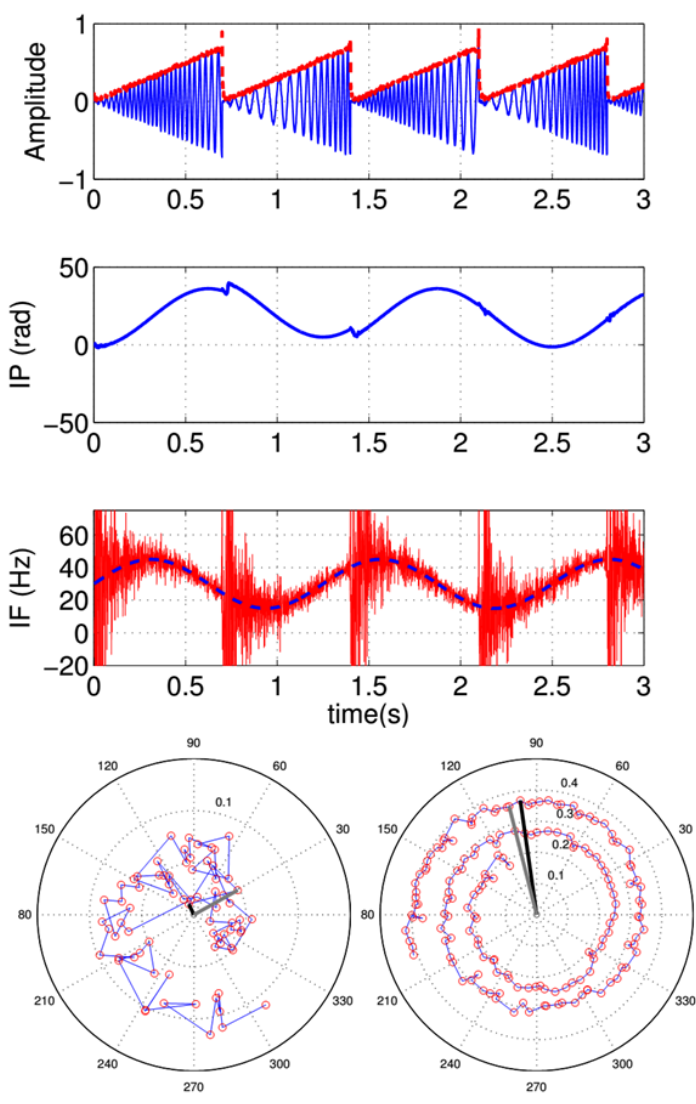

JAMSAT

Figure 1. Top row: A synthetic signal and its analytical form envelope in dashed lines; Second row: The instantaneous phase; third row: The estimated instantaneous frequency (first order time derivative of instantaneous phase in equation 3) and the true instantaneous frequency in dashed lines; Last row: The phasor representation of the analytical form during low amplitude (left) and (high amplitude) segments of the signal. The two lines starting from the origin, indicate two successive phasors in both cases. The random phase jumps and instantaneous frequency errors in low analytical signal amplitudes are notable. 
The LAAS can be considered as a major source of fake variations in phase of EEG signals. Because of background spontaneous EEG, as depicted in Figure 2, IP signal derived from the analytic form of EEG signal is prone to contain fake jumps and spikes at time instants that the analytic narrow-band signal amplitude has a low value $[39,41,47,40,48]$.

Some robust findings with regard to the underlying mathematical aspects of LAAS problem are presented in [39] and [41]. Accordingly, since the majority of jumps and spikes detected in phase of cerebral signals are susceptible to be generated or elevated by the LAAS and are not related to the brain activity, further considerations are required. Nevertheless, this notorious problem has been under-attended or ignored in earlier studies.

\section{Material and Methods}

To investigate the potential effects of LAAS on phase related quantities and discuss its consequences in clinical applications, first, a diversity of popular phase related quantities are calculated. Accordingly, the effects of LAAS on phase signals are ignored, i.e. potential fake jumps and variations are detected and then discarded, and once again similar phase quantities are calculated and compared with the previous ones. In this way, possible effects of LAAS will be shown. In the following, first the traditional phase estimation procedure, utilized to calculate phase quantities, is detailed. Furthermore, a simple procedure is suggested to discard the effects of LAAS and eventually, the popular phase related quantities are elaborated to further inspect the influence of LAAS on their ambiguity.

\section{Phase estimation procedure}

In order to estimate the phase signal by the aforementioned conventional method, a FIR filter with passband, band-width and transition band-width equal to $\mathrm{BW}=\mathrm{TB}=0.5 \mathrm{~Hz}$, maximum pass-band ripple of $\mathrm{PRP}=1$ $\mathrm{dB}$ and minimum stop-band attenuation of $\mathrm{SRP}=50 \mathrm{~dB}$ is used. Thereby, Hilbert transform is applied to the narrowbanded signal to form the analytic representation and calculate the instantaneous EEG parameters, i.e. IP and IA, using equations [1-3].

\section{LAAS-D phase estimation}

To show the effects of LAAS, the traditional phase estimation method is reused with a minor change in procedure where an additional step has beenwas added to dis-
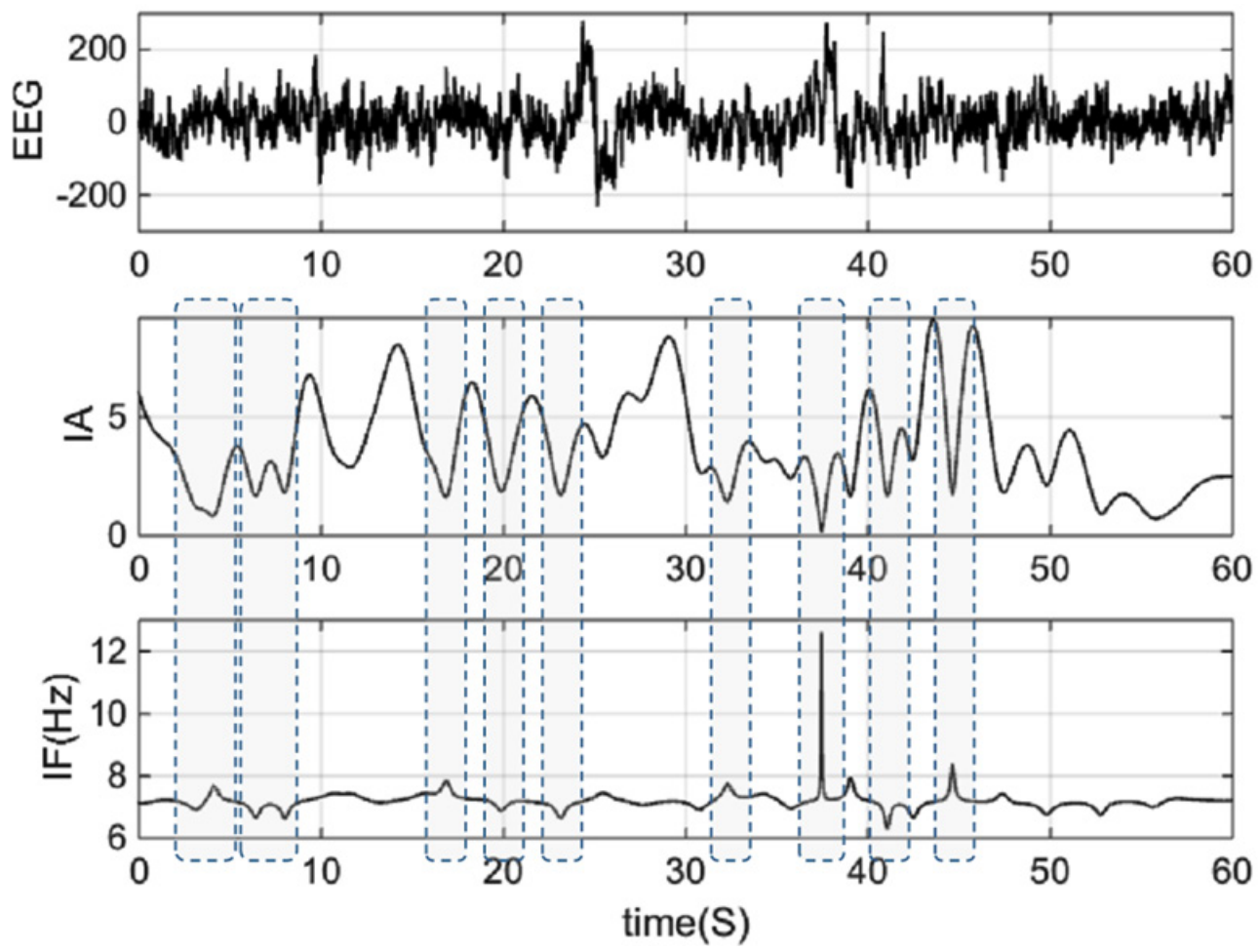

JAMSAT

Figure 2. Top panel: A raw $60 \mathrm{~s}$ EEG signal used for phase estimation. Middle panel: The Instantaneous Amplitude (IA) derived from analytic form of signal. Bottom panel: The phase derivative, i.e. instantaneous frequency, of $7 \mathrm{~Hz}$ captured from the phase sequence extracted through the analytic signal 
Table 1. Popular phase-related quantities, including: 1-Phase Derivative (PD) or Instantaneous Frequency (IF) in single channel mode, 2- Phase Shift (PS), 3- Phase Lock (PL), 4- Phase Resetting (PR) and 5- Phase-locking Value (PLV). The first three rows are clustered as Group one and PLV is considered as Group Two. Th is threshold value for calculating phase shift events (red dashed-lines in Figure 3)

\begin{tabular}{|c|c|c|}
\hline & Single Channel $[\boldsymbol{x}(\boldsymbol{n})]$ & Multi Channel $[\boldsymbol{x}(\boldsymbol{n}), \boldsymbol{y}(\boldsymbol{n})]$ \\
\hline Phase Difference (PD) & $P D_{n}=\Delta \emptyset_{n}=\emptyset_{n}-\emptyset_{n-1}$ & $P D_{n}^{x y}=\Delta \emptyset_{n}^{x y}=\emptyset_{n}^{x}-\emptyset_{n}^{y}$ \\
\hline Phase Shift (PS) & $\forall n: P S_{n}=\left\{\begin{array}{rr}1, & \Delta \emptyset_{n} \geq t h \\
0, & o . w .\end{array}\right.$ & $\forall n: P S_{n}^{x y}=\left\{\begin{array}{rr}1, & \Delta \emptyset_{n}^{x y} \geq t h \\
0, & \text { o.w. }\end{array}\right.$ \\
\hline Phase Lock (PL) & $\forall n: P L_{n}=\left|1-P S_{n}\right|$ & $\forall n: P S_{n}^{x y}=\left|1-P S_{n}^{x y}\right|$ \\
\hline PLV & \multicolumn{2}{|c|}{$\left|\frac{1}{N} \sum_{n=1}^{N} \exp \left(j\left[\emptyset_{n}^{x}(f)-\emptyset_{n}^{y}(f)\right]\right)\right|$} \\
\hline
\end{tabular}

card the effects of LAAS. For this purpose, after forming the analytic representation, the LAAS time instants are detected. Accordingly, the corresponding phase values in estimated phase sequence, i.e. concurrent with detected time instants, are simply discarded. Herein, the phase and its related quantities captured in this way are called LowAmplitude Analytic Signal Discarded (LAAS-D)phase information. A simple procedure to calculate the LAAS-D phase is elaborated in Figure3.

The threshold value Th depends on the utilized dataset, the intended application and the phase extraction procedure itself. Thus, Th is completely subjective and has to be chosen empirically.
Phase-related quantity measurements and quantifying the LAAS effects

The next step is to calculate the phase features through both the conventional phase estimation method and its LAASdiscarded version. The most popular phase-related quantities investigated here are: 1 Phase Derivative (PD) or Instantaneous Frequency (IF) in single channel mode, 2 Phase Shift (PS), 3 Phase Lock (PL), 4 Phase Resetting (PR) and 5 Phaselocking Value (PLV). Studied quantities are divided into two separate groups based on their definitions and application of interest. Table 1 illustrates the mathematical definitions of utilized phasequantities in single and multi-channel modes. In order to make the results reproducible, an EEG phase

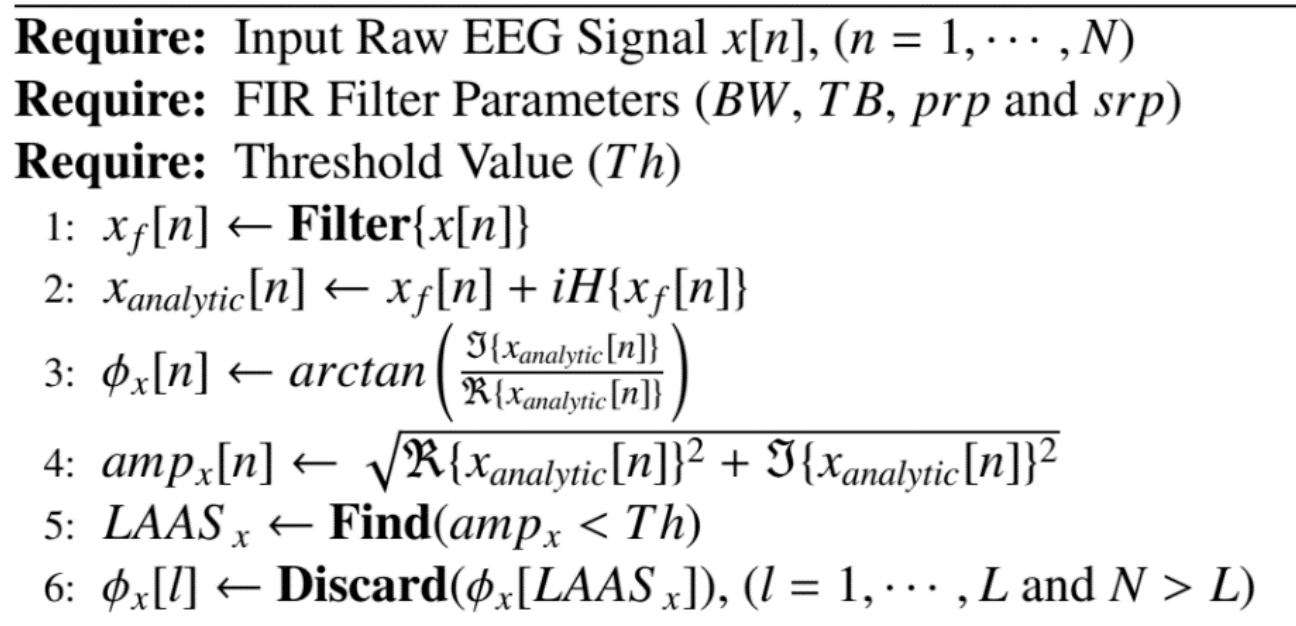


analysis toolbox related to this study, providing functions for estimating IP and IA of EEG signal and also various functions for measuring aforementioned phase related features, is available online in the Open-Source Electrophysiological Toolbox (OSET) [49, 50].

\section{Phase-related quantities group one}

Phase Derivative (PD) is one of most common and wellknown phase-related quantities used in analyzing the phase of cerebral signals. It can be calculated both in single and multichannel modes as represented in Table 1. In single channel case, for a signal $\mathrm{x}(\mathrm{t})$, the PDs is computed as firstorder time derivative of the phase sequence and i proportional to instantaneous frequency of $\mathrm{x}(\mathrm{t})$. For the continuous time signals:

$$
P D_{s}(t)=\frac{d \varnothing_{x}(t)}{d t}
$$

where the $\emptyset_{x}(t)$ is the instantaneous phase sequence of $x(t)$ captured at frequency $\mathrm{f}$. In multi-channel case, $\mathrm{PD}_{\mathrm{m}}$ is calculated as the phase difference between two signals $\mathrm{x}(\mathrm{t})$ and $y(t)$ as below:

$$
P D_{m}(t)=\varnothing_{x}(t)-\emptyset_{y}(t)
$$

We simply use the notation PD for both single and multichannel cases.

\section{Phase Shift (PS)}

Phase shift events are generated by a significant displacement between the phase sequences of two signals (multichannel) or a significant abrupt increase or decrease in phase of a signal (single channel). As represented in Table 1, calculating the PS events consists of, first detecting the phasedisplacements or phase-jumps, and then choosing the most significant ones as the PS events [30, 51, 52]. Accordingly, the first step in computing the phase shift events for continuous time signals is to calculate the $\mathrm{d}(\mathrm{t})$ as,

$$
d(t)=\frac{d(P D(t))}{d t}
$$

and then the PS can be obtained as follows:

$$
\forall t: P S=d(t) \mid d(t) \geq t h
$$

where Th is the threshold used to discriminate between significant and non-significant displacements or jumps. Accordingly, the times t corresponding to PS components obtained from Equation above demonstrate the phase shift events.

\section{Phase Lock (PL)}

Phase locked signals have two equal or closely related phase sequences. In the case of spontaneous or ongoing cerebral signals where there is no evoking stimulus, a near zero first derivative of PD demonstrates phase lock [30, 51, 52]. In other words, PL is the counterpart of PS. Therefore, after calculating the $\mathrm{d}(\mathrm{t})$ as represented in previous page, the PL can be computed as follows:

$$
\forall t: P L=d(t) \mid d(t) \leq t h
$$

where similarly, the times t corresponding to PL components obtained from Equation above shows the phase lock events.

\section{Phase Resetting (PR)}

Basically, phase resetting is made up of a phase shift followed by a phase difference stability, i.e. phase lock [53]. Thus, each pair of PS and PL, starting from the beginning of the PS and finishing by the end of PL (beginning of the next PS), is called a phase reset. Figure 4 shows the PR, PL, and PS events calculated for a signal.

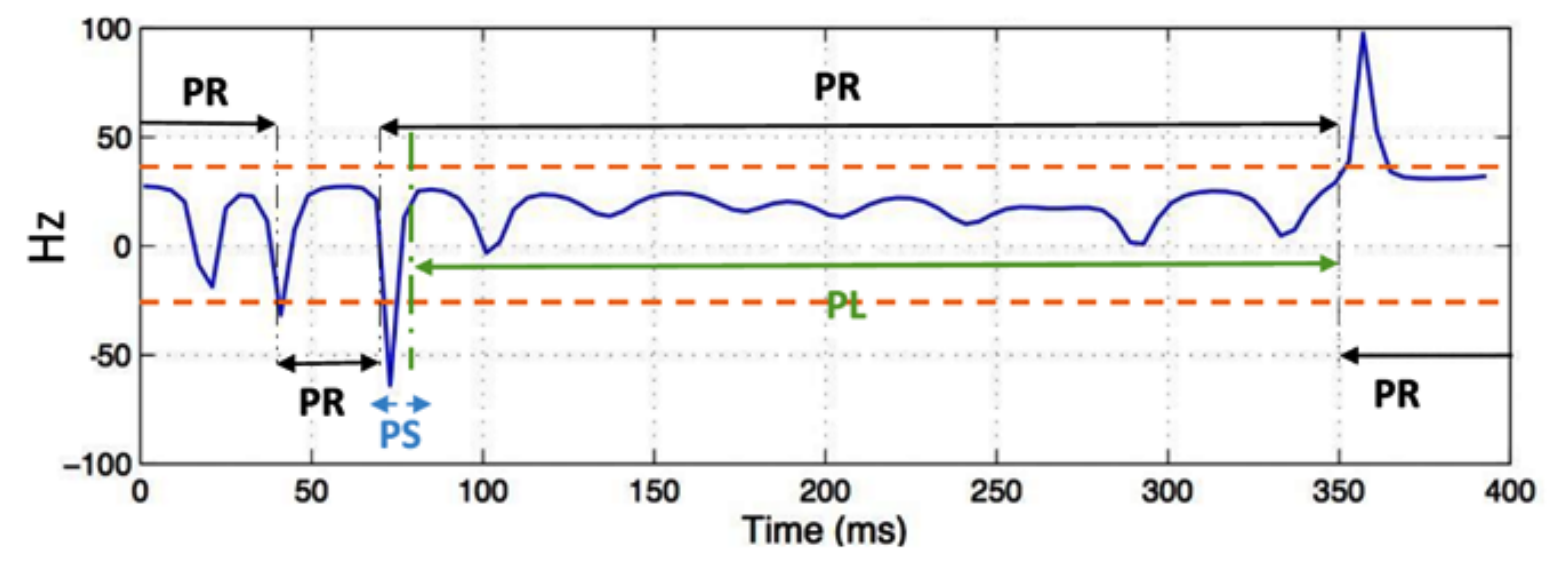

JAMSAT

Figure 4. Phase Resetting (PR), Phase Lock (PL) and Phase Shift (PS) events captured from a $400 \mathrm{~ms}$ segment signal. The dashed red lines show the assumed threshold (Th) for measuring PS. 


\section{Phase-related quantities group two}

Phase-locking Value (PLV) is one of most common quantities used in analyzing phase of a signal which describes how much the difference between phases of two signals is constant. In order to calculate PLV, there are three main steps summarized as follow [43, 54]: 1 Calculating a sequence consisting instantaneous frequency-specific phase values within a temporal window, 2Determining the instantaneous phase-differences (in frequency $f$ and time $t$ ), and 3 Quantifying the local stability of this phase-differences across trials of cerebral signals.

Accordingly, the stability of phase-differences between signals, $x(t)$ and $y(t)$, can be quantified as below [43]

$$
\left.\operatorname{PLV}(\mathrm{f})=\mid 1 / T \sum_{t=1}^{T} \exp (j(t, f)-\varnothing y(t, f))\right) \mid
$$

where $\mathrm{T}$ is the length of windowed trials. PLV varies between 0 and 1, where 0 indicates two completely non-synchronized signals and 1 shows a complete synchronization $[43,54]$

In order to investigate the effects of LAAS on PLV measurements, the PLV values are calculated between each pair of EEG channels (i.e. pairwise PLV) in a desired frequency $f$ and stored in PLV matrix PLVf as below [11]:

$$
P L V=\left[\begin{array}{ccccc}
P l V_{11} & P l V_{12} & \cdot & \cdot & P l v_{1 j} \\
P l V_{21} & P l V_{22} & \cdot & \cdot & P l v_{2 j} \\
\cdot & \cdot & \cdot & \cdot & \cdot \\
\cdot & \cdot & \cdot & \cdot & \cdot \\
P l V_{i 1} & P l V_{i 2} & \cdot & \cdot & P l v_{i j}
\end{array}\right.
$$

where $i$ and $j$ vary with number of EEG channels (PLVnxn in n-channel EEG signal). Each value PLVij shows the calculated PLV between channels i-th and j-th in intended frequency f. In this study, PLV values for different frequencies within alpha and beta bands are calculated

\section{Quantifying the PLV variations due to LAAS}

The Magnitude Squared Coherence (MSC) is calculated as a reference quantity to detect PLV variations due to LAAS. The underlying reason is that, MSC and phase synchronization (i.e. PLV) provide essentially the same information. As reported in previous studies complete synchrony is manifested by highly coherent phases [55]. The conventional approach for computing MSC is based on weighted windowing of the Fourier Transform (FT) of signals [56, 58]. Assuming $\mathrm{x}(\mathrm{t})$ and $\mathrm{y}(\mathrm{t})$ to be two random processes (two EEG channels in this study), the MS Cf can be obtained using the power spectral densities in frequency f through Equation [56].

$$
C_{x y}=\left|M S C_{f}\right|^{2}=\frac{\left|P S D_{x y}(f)\right|^{2}}{P S D_{x x}(f) P S D_{y y}(f)}
$$

Similar to PLV, MSC varies between 0 and 1 , where $\mathrm{MSC}_{\mathrm{f}}=1$ denotes highly coherent signals in frequency of $\mathrm{f}$ and vice versa. Accordingly, $\mathrm{MSC}_{\mathrm{f}}$ is calculated between each possible pair of EEG channels in chosen frequencies, and the results are stored in a matrix similar to PLVf in Equation 11. Accordingly, correlation indices are used to quantify the relations between PLV and MSC matrices. To this end, two different correlations indices, namely Pearson linear correlation and Spearman rank-order (nonlinear) correlation, are employed. The Pearson correlation evaluates the strength of a linear association between two variables. The Spearman correlation evaluates the monotonic relationship between two variables. In a monotonic relation, the variables tend to change together, but not necessarily at a constant rate.

In this study, Pearson and Spearman correlations are used to detect both linear and nonlinear associations between PLV and MSC matrices. As such, the correlation results can reveal the possible effects of low-amplitude analytic signals in PLV measurements.

\section{Results}

Our present dataset includes 5 trials of 128channel EEG recordings of 4 different subjects recorded when submitted to visual stimuli. Steady-state Visual Evoked Potentials (SSVEP) were sampled at $256 \mathrm{~Hz}$ and three different SSVEP stimulation frequencies are available (i.e. $8 \mathrm{~Hz}, 14 \mathrm{~Hz}$ and $28 \mathrm{~Hz}$ ). A complete description is represented in number [57]

Illustrating the LAAS effects on phase-related quantities group one

The quantities described in the first group of phase features are calculated using both the conventional phase estimation procedure and its LAAS discarded version foparticular frequencies, i.e. $8 \mathrm{~Hz}$ of alpha band. The effects of LAAS (i.e. low foreground SNR) on single channel EEG phase parameters is illustrated in Figure 5. Despite the first 3 seconds where the estimations are affected by the transient effect of FIR filter in frequency filtering, as depicted, only 2 out of 28 detected phase shift events, are not concurrent with low values of foreground amplitude and possibly have physiological origins. According to Figure 5, a significant part of the detected phase shift events from cerebral signals are not related to the brain activity and are just merely side effects of the utilized conventional phase estimation procedure. 

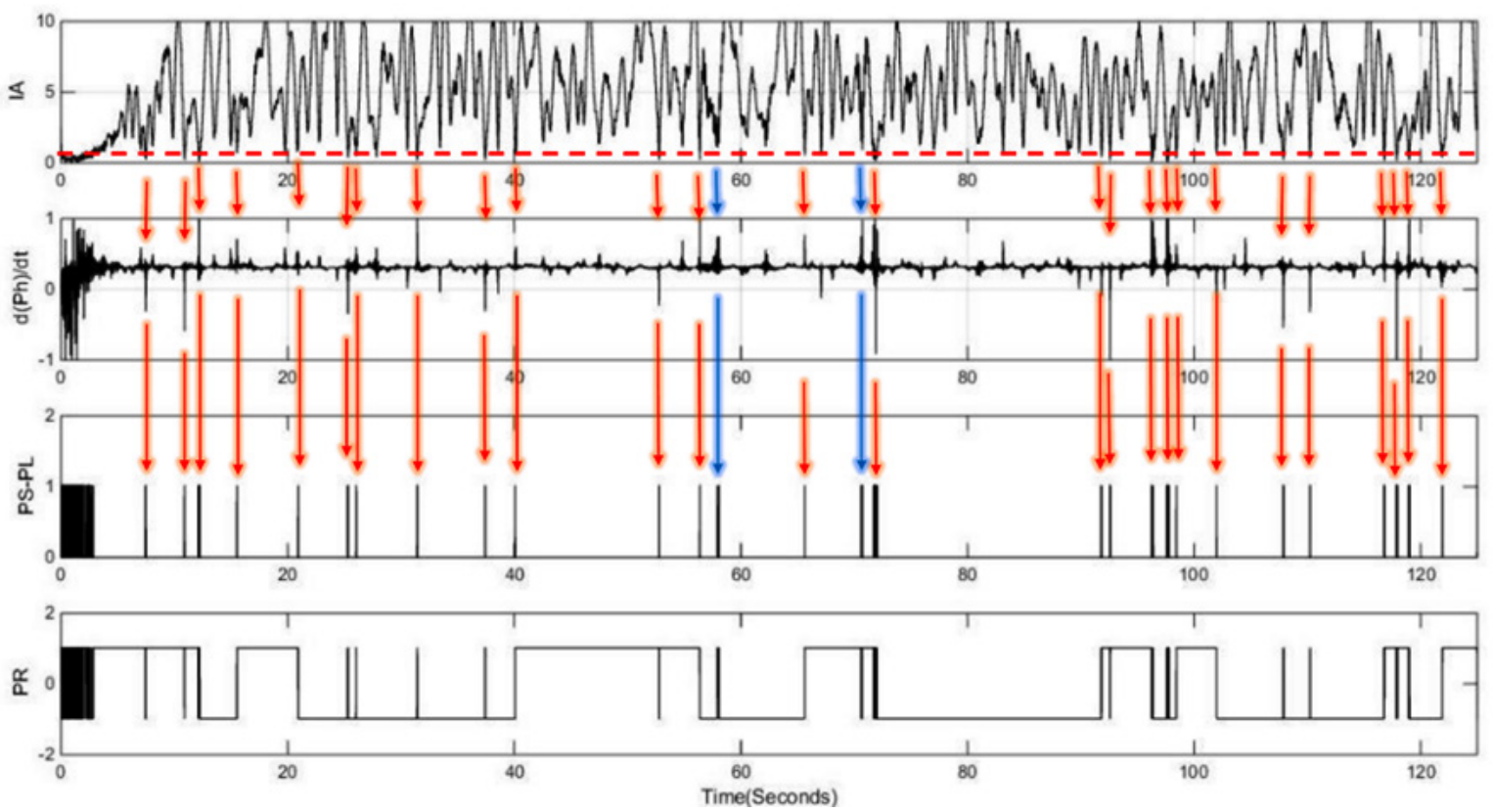

JAMSAT

Figure 5. The low-amplitude analytic signal effects on first group of phase-related quantities in a single-channel EEG recording. Panels demonstrate instantaneous amplitude, phase difference, phase shift (lock) and phase resetting events from top to bottom, respectively. The red dashed-line represents a threshold for detecting low values of amplitude. The phase shift events concurrent with LAAS (red arrows) are notable

For multi-channel investigation, two EEG channels of a recording in introduced dataset are used. Results are summarized in Figure 6. As illustrated, similarly, the LAAS effects on phase quantities are undeniable and phase resetting pat- terns significantly changed after discarding the phase values concurrent with LAAS time instants. Therefore, once again, we encountered that physiological information embedded in the EEG phase highly affected by side effects of the con-

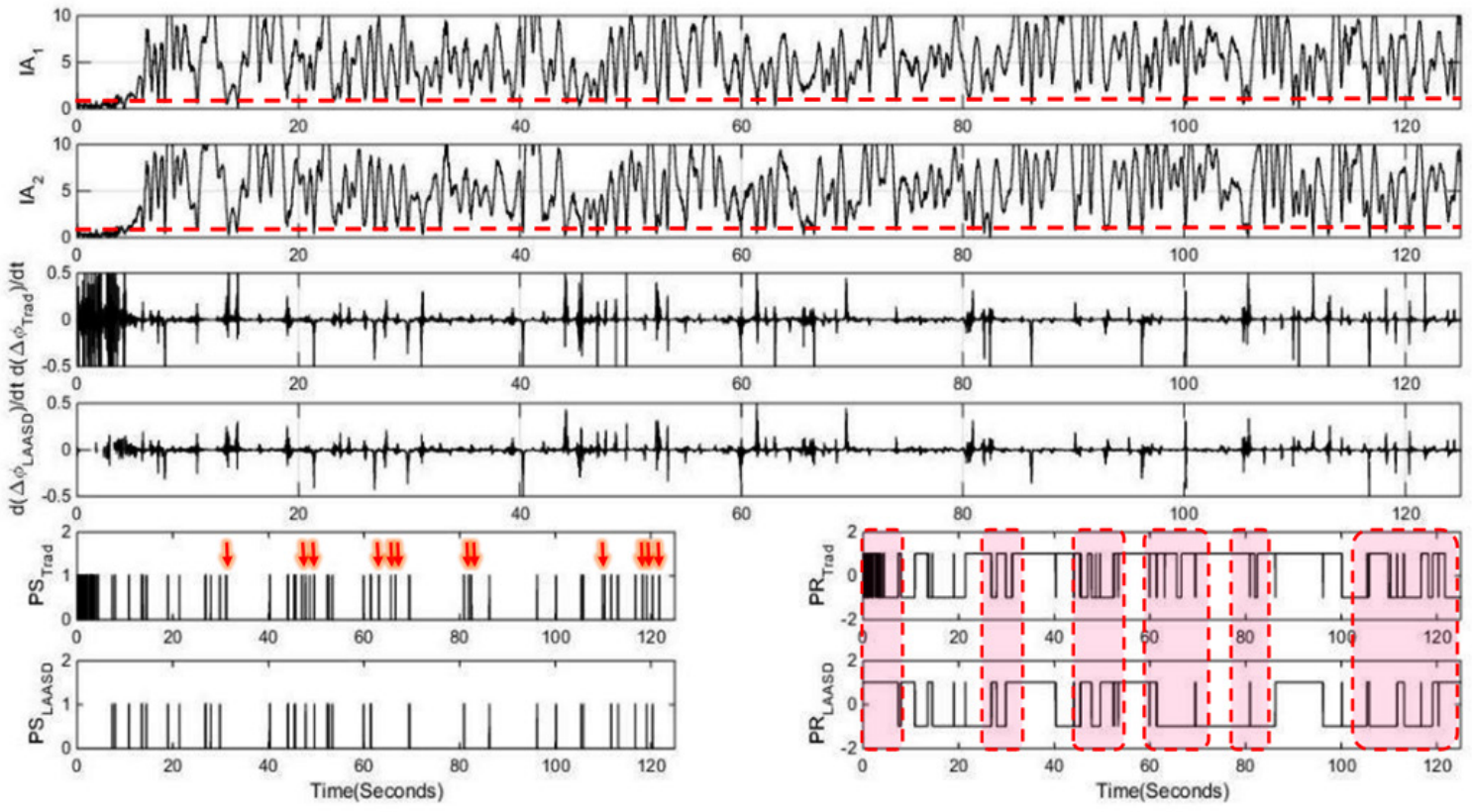

JAMSAT

Figure 6. The low-amplitude analytic signal effects on first group of phase-related quantities in multi-channel EEG recording. Panels show instantaneous amplitudes of both channels (first and second), multi-channel PDs of conventional and LAAS-D phase estimates (third and fourth) and their corresponding PS events (bottom left side) and PR (bottom right side). Notice the change in PR patterns after discarding the phase values concurrent with LAAS time instants. 
ventional and widely-used phase estimation procedure. Consequently, interpretations such as associating phase resetting and phase shift to cognitive responses in brain would require further investigations for the proof of integrity.

\section{Illustrating the LAAS effects on phase-related quantities group two}

For this part, the results of a new robust phase estimation method, namely Transfer Function Perturbation (TFP) phase estimation method, proposed in [41] is also presented and compared with conventional method for further discussions. In this Monte Carlo estimation based method, the quality of phase measure is improved through generating random ensembles of IP by perturbing the estimations parameters with infinitesimal variations. TFP also modifies the filtering procedure and tools (e.g. IIR zero-phase forward-backward filtering) to prevent information leak of EEG phase caused by filtering step. A complete description of TFP method is provided in our earlier study [41]. Herein, we use a function generated by the authors of a recent report [41], to implement the TFP method provided in a related report [50].

The linear and nonlinear correlation results for pairwise synchronizations over cortical records of three subjects in employed dataset are calculated through aforementioned EEG phase estimation methods with respect to three different frequency components in alpha and beta rhythms (i.e. 8, 14, and $28 \mathrm{~Hz}$ ) and are illustrated in Figures 7 and 8, respectively.

The differences between results of different methods, namely, TFP, conventional phase estimation method and its LAAS discarded version, should be highlighted. As an observation made here, interpretations of phase synchrony (one of the most important indexes in assessing cerebral connectivity) in cortical records is highly dependent on phase estimation procedure.

\section{Discussion}

The focus of current study was discussing the reliability of EEG phase information in cognitive interpretations. The impact of this investigation within the neuroscience community mostly includes applications such as assessing connectivity within the brain, brain cognitive response studies and $\mathrm{BCI}$ systems which widely employ phase information embedded in the cortical brain records.
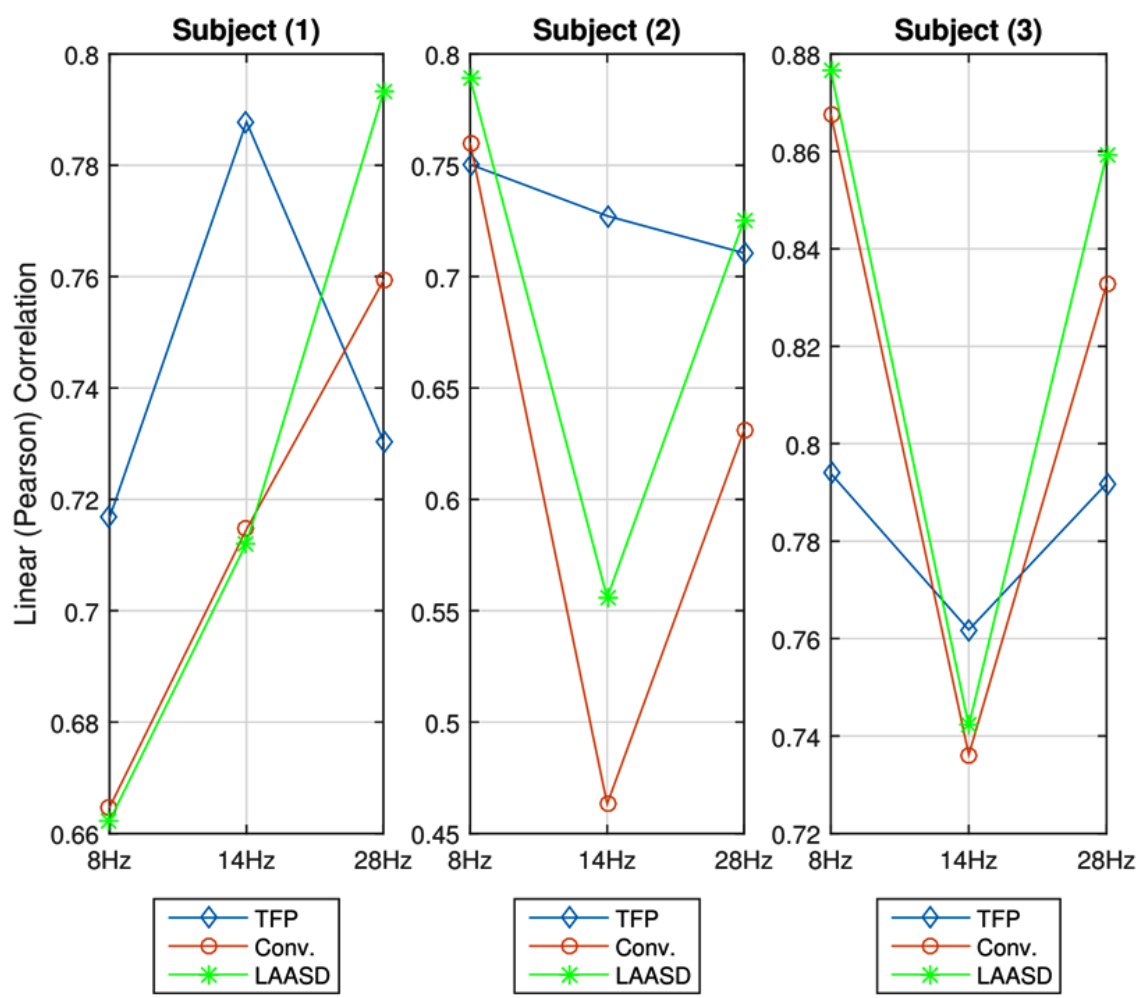

JAMSAT

Figure 7. The linear (Pearson) correlation results for pairwise synchronizations over cortical records of three subjects in employed dataset. Notice the differences between different methods. Interpretation of phase synchrony in cortical records is highly dependent to the phase estimation procedure. 


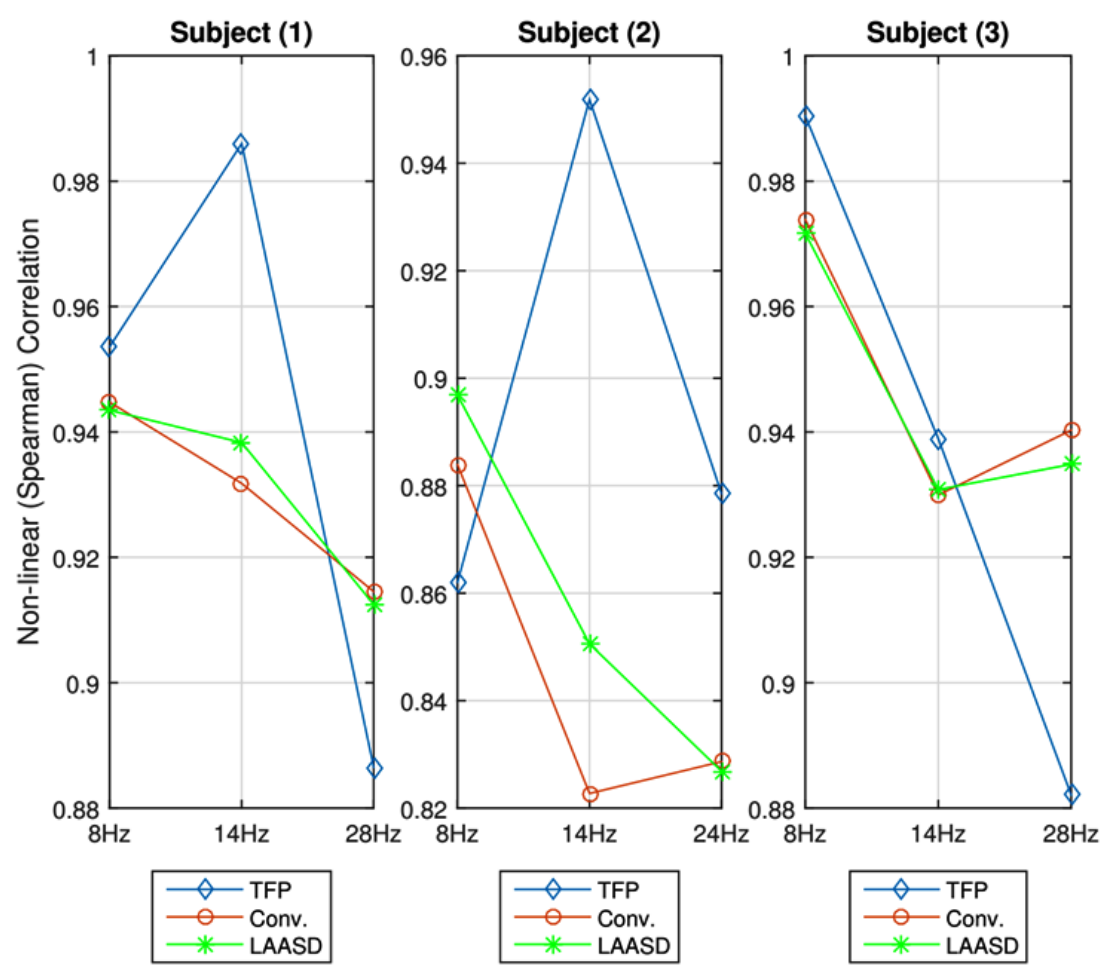

JAMSAT

Figure 7. The nonlinear (Spearman) correlation results for pair wise synchronizations over cortical records of three subjects in employed data set. Notice the differences between different methods. Interpretation of phase synchrony in cortical records is highly dependent on the phase estimation procedure.

Instantaneous phase estimation in low foreground SNR (low amplitudes of desired activity), is prone to be unreliable due to background spontaneous EEG noise [39]. It has been reported that phase values in time instants concurrent with low values of analytic amplitude are unreliable and cannot be linked to physiological origins within brain [39, 41]. As discussed, the LAAS effects on calculating the phase-related quantities are inevitable [39, 41].

Herein, we proposed a simple approach to inspect the effects of LAAS on calculating phase related features and illustrate their potential impact on clinical interpretations using these quantities. The results showed that, the information in EEG phase is significantly affected by LAAS problem and a majority of variations in phase quantities are merely side effects of adopted phase estimation procedure. Therefore, physiological interpretations previously proposed using these quantities require more investigations for proof of integrity.

\section{Conclusions}

The current study reveals that interpreting the cerebral signals' phase information without considering the am- plitude can lead to unreliable and incorrect clinical results. For instance, studying the brain's epileptic activity using EEG phase information [20, 21], or interpreting the brain's different cognitive states as in some earlier studies [27, 28, 29, 17, 30, 31, 32, 33, 34] are very likely to be affected by LAAS and phase estimation procedures. Perhaps, this could be the underlying reason of opposite results reported in mentioned references.

As discussed, one way of preventing this ambiguity is to consider the amplitude of foreground desired signal alongside with the estimated phase. For this purpose, a statistical approach is proposed [39] where confidence intervals based on analytic envelope are computed for IP measures and the variations of phase quantities within these intervals are considered normal (as a random variable) and unrelated to the brain activity. This approach can significantly increase the quality of clinical interpretations using the EEG phase information. Moreover, another approach may include using robust cerebral phase estimation methods such as TFP [41] to enhance the quality of phase signals and reduce the side effects of estimation procedures. 


\section{Acknowledgments}

This research was supported by the Cognitive Sciences and Technologies Council of Iran (COGC), under the grant number 2250. Author would like to thank Dr. R. Sameni for his insightful comments.

\section{Conflict of Interest}

The author declared no Conflict of Interests.

\section{References}

[1] Gray CM, Konig P, Engel AK, Singer W, et al. Oscillatory responses in cat visual cortex exhibit inter-columnar synchronization which reflects global stimulus properties Nature. 1989; 338(6213):334-337. doi: 10.1038/338334a0

[2] Marshall W. Statistical analysis of EEG phase shift events [PhD thesis]. Waterloo: University of Waterloo; 2014

[3] Krusienski DJ, McFarland DJ, Wolpaw JR. Value of amplitude, phase, and coherence features for a sensorimotor rhythm-based brain-computer interface. Brain Research Bulletin. 2012; 87(1):130-134. doi: 10.1016/j.brainresbull.2011.09.019

[4] He W, Wei P, Zhou Y, Wang L. Combination of amplitude and phase features under a uniform framework with EMD in EEG-based brain-computer interface. Paper presented at: The Annual International Conference of the IEEE Engineering in Medicine and Biology Society. 2012 Aug 28-1; San Diego, USA.

[5] Townsend G, Feng Y. Using phase information to reveal the nature of event-related desynchronization. Biomedical Signal Processing and Control. 2008; 3(3):192-202. doi: 10.1016/j.bspc.2008.01.003

[6] Razavipour F, Sameni R. A study of event related potential frequency domain coherency using multichannel electroencephalogram subspace analysis. Journal of Neuroscience Methods. 2015; 249:22-28. doi: 10.1016/j.jneumeth.2015.03.037

[7] Picton TW, Dimitrijevic A, John MS, Van Roon P. The use of phase in the detection of auditory steady-state responses. Clinical Neurophysiology. 2001; 112(9):1698-1711. doi: 10.1016/s1388-2457(01)00608-3

[8] De Tommaso M, Marinazzo D, Guido M, Libro G, Stramaglia S, Nitti L, et al. Visually evoked phase synchronization changes of alpha rhythm in migraine: Correlations with clinical features. International Journal of Psychophysiology. 2005; 57(3):203-210. doi: 10.1016/j.ijpsycho.2005.02.002

[9] Sakkalis V. Review of advanced techniques for the estimation of brain connectivity measured with EEG/MEG. Computers in Biology and Medicine. 2011; 41(12):11101117. doi: 10.1016/j.compbiomed.2011.06.020
[10] Friston KJ. Functional and effective connectivity: A review. Brain Connectivity. 2011; 1(1):13-36. doi: 10.1089/ brain. 2011.0008

[11] Gomez-Herrero G. Brain connectivity analysis with EEG [PhD thesis]. Tampere: Tampere University of Technology; 2010.

[12] Sauseng P. Brain oscillations: Phase-locked EEG alpha controls perception. Current Biology. 2012; 22(9):R306R308. doi: 10.1016/j.cub.2012.03.029

[13] Fell J, Axmacher N. The role of phase synchronization in memory processes. Nature reviews neuroscience. 2011 12(2):105-118. doi: 10.1038/nrn2979

[14] Siegel M, Warden MR, Miller EK. Phase-dependent neuronal coding of objects in short-term memory. Proceedings of the National Academy of Sciences. 2009; 106(50):2134121346. doi: $10.1073 /$ pnas.0908193106

[15] Kaminski M, Blinowska KJ. A new method of the description of the information flow in the brain structures Biological Cybernetics. 1991; 65(3):203-210. doi: 10.1007/ bf00198091

[16] Kaminski M, Blinowska K, Szelenberger W. Topographic analysis of coherence and propagation of EEG activity during sleep and wakefulness. Electroencephalography and clinical Neurophysiology. 1997; 102(3):216-227. doi: 10.1016/s0013-4694(96)95721-5

[17] Thatcher RW, North DM, Biver CJ. Development of cortical connections as measured by EEG coherence and phase delays. Human Brain Mapping. 2008; 29(12):1400-1415. doi: $10.1002 / \mathrm{hbm} .20474$

[18] Nunez PL, Srinivasan R. Electric fields of the brain: The neurophysics of EEG. 2nd edition. Oxford: Oxford University Press; 2006.

[19] Suzuki. Phase relationships of alpha rhythm in man. The Japanese Journal of Physiology. 1974; 24(6):569-586. doi: 10.2170/ijphysiol.24.569

[20] Chavez M, Le Van Quyen M, Navarro V, Baulac M, Martinerie J. Spatio-temporal dynamics prior to neocortical seizures: Amplitude versus phase couplings. IEEE Transactions on Biomedical Engineering. 2003; 50(5):571-583. doi: $10.1109 /$ tbme.2003.810696

[21] Netoff TI, Schiff SJ. Decreased neuronal synchronization during experimental seizures. Journal of Neuroscience. 2002; 22(16):7297-7307. PMID: 12177225

[22] Stam C, Van Der Made Y, Pijnenburg Y, Scheltens P. EEG synchronization in mild cognitive impairment and Alzheimer's disease. Acta Neurologica Scandinavica. 2003; 108(2):90-96. PMID: 12859284

[23] Stam CI, van Walsum AMvC, Pijnenburg YA, Berendse HW, de Munck JC, Scheltens P, et al. Generalized synchronization of MEG recordings in Alzheimers disease: Evidence for involvement of the gamma band. Journa of Clinical Neurophysiology. 2002; 19(6):562-574. doi 10.1097/00004691-200212000-00010

[24] Thatcher RW, North DM, Neubrander J, Biver CJ, Cutler S, DeFina P. Autism and EEG phase reset deficient GABA mediated inhibition in thalamo-cortical circuits. Devel- 
opmental Neuropsychology. 2009; 34(6):780-800. doi: $10.1080 / 87565640903265178$

[25] Jeanmonod D, Magnin M, Morel A, Siegemund M, Cancro A, Lanz M, et al. Thalamocortical dysrhythmia II. Clinical and surgical aspects. Thalamus \& Related Systems. 2001; 1(03):245-254. doi: 10.1017/s1472928801000267

[26] Jeanmonod D, Schulman J, Ramirez R, Cancro R, Lanz M, Morel A, et al. Neuropsychiatric thalamocortical dysrhythmia: Surgical implications. Thalamus \& Related Systems. 2003; 2(02):103-113. doi: 10.1017/s1472928803000104

[27] Rizzuto D, Madsen J, Bromfield E, Schulze-Bonhage A, Seelig D, Aschenbrenner-Scheibe R, et al. Reset of human neocortical oscillations during a working memory task. Proceedings of the national academy of Sciences. 2003; 100(13):7931-7936. doi: 10.1073/pnas.0732061100

[28] Tallon-Baudry C, Bertrand O, Fischer C. Oscillatory synchrony between human extrastriate areas during visual short-term memory maintenance. The Journal of Neuroscience. 2001; 21(20):177. PMID: 11588207

[29] Squire LR. Mechanisms of memory. Science. 1986; 232(4758):1612-1619. doi: PMID: 3086978

[30] Thatcher RW, North D, Biver C. Intelligence and EEG phase reset: A two compartmental model of phase shift and lock. NeuroImage. 2008; 42(4):1639-1653. doi: 10.1016/j.neuroimage.2008.06.009

[31] Sauseng P, Klimesch W, Gruber WR, Birbaumer N. Crossfrequency phase synchronization: A brain mechanism of memory matching and attention. Neuroimage. 2008; 40(1):308-317. doi: 10.1016/j.neuroimage.2007.11.032

[32] Cosmelli D, David O, Lachaux JP, Martinerie J, Garnero L, Renault B, et al. Waves of consciousness: Ongoing cortical patterns during binocular rivalry. Neuroimage. 2004; 23(1):128-140. doi: 10.1016/j.neuroimage.2004.05.008

[33] Varela F, Lachaux JP, Rodriguez E, Martinerie J. The brainweb: Phase synchronization and large-scale integration. Nature Reviews Neuroscience. 2001; 2(4):229-239. doi: $10.1038 / 35067550$

[34] John ER. The neurophysics of consciousness. Brain Research Reviews. 2002; 39(1):1-28. doi: 10.1016/s01650173(02)00142-x

[35] John ER. From synchronous neuronal discharges to subjective awareness? The Boundaries of Consciousness: Neurobiology and Neuropathology. 2005; 150(1):143-593. doi: 10.1016/s0079-6123(05)50011-6

[36] Vaadia E, Haalman I, Abeles M, Bergman H, Prut Y, Slovin $\mathrm{H}$, et al. Dynamics of neuronal interactions in monkey cortex in relation to behavioural events. Nature. 1995; 373(6514):515-518. doi: 10.1038/373515a0

[37] Sauseng P, Klimesch W. What does phase information of oscillatory brain activity tell us about cognitive processes? Neuroscience \& Biobehavioral Reviews. 2008; 32(5):10011013. doi: 10.1016/j.neubiorev.2008.03.014

[38] Kahana MJ. The cognitive correlates of human brain oscillations. The Journal of Neuroscience. 2006; 26(6):1669-72. doi: 10.1523/jneurosci.3737-05c.200
[39] Sameni R, Seraj E. A robust statistical framework for instantaneous electroencephalogram phase and frequency analysis [Internet]. 2016 [Cited 2016 Aug 23]. Available from: https://hal.archives-ouvertes.fr/hal-01355465/ document.

[40] Chavez M, Besserve M, Adam C, Martinerie J. Towards a proper estimation of phase synchronization from time series. Journal of Neuroscience Methods. 2006; 154(1-2):149160. doi: 10.1016/j.jneumeth.2005.12.009

[41] Seraj E, Sameni R. Robust electroencephalogram phase estimation with applications in brain-computer interface systems; 2016. [Cited 2016 Oct 10]. Available from: https://hal.archives-ouvertes.fr/hal-01378726/

[42] Mormann F, Lehnertz K, David P, Elger CE. Mean phase coherence as a measure for phase synchronization and its application to the EEG of epilepsy patients. Physica D: Nonlinear Phenomena. 2000; 144(3):358369. doi: 10.1016/ s0167-2789(00)00087-7

[43] Lachaux JP, Rodriguez E, Martinerie J, Varela FJ, et al. Measuring phase synchrony in brain signals. Human Brain Mapping. 1999; 8(4):194-208. doi: PMID: 10619414

[44] Le Van Quyen M, Foucher J, Lachaux JP, Rodriguez E, Lutz A, Martinerie J, et al. Comparison of Hilbert transform and wavelet methods for the analysis of neuronal synchrony. Journal of Neuroscience Methods. 2001; 111(2):83-98. doi: 10.1016/s0165-0270(01)00372-7

[45] Cohen L, Loughlin P, Vakman D. On an ambiguity in the definition of the amplitude and phase of a signal. Signal Processing. 1999; 79(3):301-307. doi: 10.1016/s01651684(99)00103-6

[46] Oliveira PM, Barroso V. Instantaneous frequency of multicomponent signals. IEEE Signal Processing Letters. 1999; 6(4):81-83. doi: 10.1109/97.752060

[47] Picinbono B. On instantaneous amplitude and phase of signals. IEEE Transactions on Signal Processing. 1997; 45(3):552-560. doi: 10.1109/78.558469

[48] Freeman WJ. Origin, structure, and role of background EEG activity. Part 2. Analytic phase. Clinical Neurophysiology. 2004; 115(9):2089-2107. doi: 10.1016/j. clinph.2004.02.028

[49] Sameni R. [The open-source electrophysiological toolbox (OSET) [Internet]. 2010 [Cited 2010 Jan 10]. Available from: https:/ / www.researchgate.net/publication/235325036_ The_Open-Source_Electrophysiological_Toolbox_OSET_ version_21

[50] Seraj E. Cerebral Signal Phase Analysis Toolbox-User Guide. Computers in Biology and Medicine [Internet]. 2016 [Cited 2016 Oct 07]. Available from: https://www. sparrho.com/item/cerebral-signal-phase-analysis-toolbox-user-guide/9c7f46/

[51] Thatcher RW, North DM, Biver CJ. Self-organized criticality and the development of EEG phase reset. Human Brain Mapping. 2009; 30(2):553574. doi: 10.1002/hbm.20524

[52] Freeman WJ, Burke BC, Holmes MD. Aperiodic phase resetting in scalp EEG of beta-gamma oscillations by state transitions at alpha-theta rates. Human Brain Mapping. 2003; 19(4):248-272. doi: 10.1002/hbm.10120 
[53] Pikovsky A, Rosenblum M, Kurths J. Synchronization: A universal concept in nonlinear sciences. Cambridge Nonlinear Sciences Series 12. Cambridge: Cambridge University Press; 2003.

[54] Rosenblum MG, Pikovsky AS, Kurths J. Phase synchronization of chaotic oscillators. Physical Review Letters. 1996; 76(11):1804-7. doi: 10.1103/physrevlett.76.1804

[55] Mezeiova K, Palus M. Comparison of coherence and phase synchronization of the human sleep electroencephalogram. Clinical Neurophysiology. 2012; 123(9):1821-1830. doi: 10.1016/j.clinph.2012.01.016

[56] Carter G, Knapp C, Nuttall A. Estimation of the magnitude-squared coherence function via overlapped fast Fourier transform processing. IEEE transactions on audio and electroacoustics. 1973; 21(4):337-344. doi: 10.1109/ tau.1973.1162496

[57] Bakardjian H, Tanaka T, Cichocki A. Optimization of SSVEP brain responses with application to eight-command brain-computer interface. Neuroscience Letters. 2010; 469(1):34-38. doi: 10.1016/j.neulet.2009.11.039

[58] Seraj E. Cerebral synchrony assessment: A general review on cerebral signals' synchronization estimation concepts and methods [Internet]. 2016 [Cited 2016 Dec 12]; Available from: https://arxiv.org/abs/1612.04295 
